\title{
Opportunities and barriers for telerehabilitation during Coronavirus outbreak
}

\author{
Parisa Arzani ${ }^{1 *}$ (D) Minoo Khalkhali Zavieh ${ }^{2}$, Khosro Khademi-Kalantari ${ }^{2}$, Alireza Akbarzadeh Baghban ${ }^{3}$ \\ Received: 8 Jun 2020 \\ Published: 4 Aug 2021
}

Conflicts of Interest: None declared

Funding: Shahid Beheshti University of Medical Sciences

\begin{abstract}
*This work has been published under CC BY-NC-SA 1.0 license.
Copyright $₫$ Iran University of Medical Sciences
\end{abstract}

Cite this article as: Arzani P, Khalkhali Zavieh M, Khademi-Kalantari Kh, Alireza Akbarzadeh Baghban. Opportunities and barriers for telerehabilitation during Coronavirus outbreak. Med J Islam Repub Iran. 2021 (4 Aug);35:100. https://doi.org/10.47176/mjiri.35.100

\section{Brief Communication}

In late December 2019, the WHO China Country Office was informed of cases of pneumonia with unknown etiology, which quickly spread from a single city to the entire country (1). After a while, it shown that a highly contagious strain of the coronavirus was spreading (2). It did not take long for this epidemic to spread globally. After that, the World Health Organization (WHO) declared this epidemic disease as a pandemic (3). Scientists believe quarantining and contact tracing of symptomatic individuals alone may not be the best way; therefore, for stopping the transmission of the virus early, strong control measures, such as social distancing, are necessary (4). The objectives of this social distancing are to decrease or interrupt the transmission of COVID-19 in a population and consequently reduce transmission, delaying the epidemic peak, reducing the size of the epidemic peak, and spreading cases over a longer time to relieve pressure on the health care system (5).

The close and direct contact of physiotherapists and other rehabilitation practitioners is an inevitable part of rehabilitation, which makes them susceptible to the transmission of infectious diseases (6). In response to the evolving COVID-19 pandemic, physical therapists and other rehabilitation practitioners must consider alternative methods of service provision, specially telerehabilitation

\footnotetext{
Corresponding author: Parisa Arzani, pa.arzani@sbmu.ac.ir

1. Department of Physiotherapy, School of Rehabilitation, Student Research Committee, Shahid Beheshti University of Medical Sciences, Tehran, Iran

2. Department of Physiotherapy, School of Rehabilitation, Shahid Beheshti University of Medical Sciences, Tehran, Iran

3. Proteomics Research Center, Department of Basic Science, School of Rehabilitation, Shahid Beheshti University of Medical Sciences, Tehran, Iran
}

services. Moreover, telerehabilitation is the delivery of health care and the exchange of health care information across distances $(7,8)$.

The coronavirus outbreak could provide a turning spot for this method of the rehabilitation area that has struggled to gain widespread acceptance. The WHO, citing its many benefits, has stated that telehealth should be one of the main parts of health care strategic plans to change health systems in the 21 st century (9) . This article aimed to outline the key requirements to ensure that the value of telerehabilitation is fully realized, not only in emergencies (such as pandemics) but also in everyday practice.

Telerehabilitation is a fast-developing area, which was first defined by the National Institute of Disability and Rehabilitation in the United States in 1997 (9). The formal history of telerehabilitation is directly tied to advancements in technology and responding to the requirements. It encompasses a considerable range of rehabilitation services that are offered in different formats including, teleconsultation, teleconferencing, telematics, tele-education, tele monitoring (interactive), telemonitoring (unobtrusive), telesupport, tele-evaluation, teleassessment, telecompliance, teletherapy, teleplay, and telecoaching (10). Telerehabilitation goals include reducing the time to diagnose the disease (11), improving patient care $(12,13)$, improving access to rehabilitation for people in rural areas

\section{$\uparrow$ What is "already known" in this topic:}

The coronavirus outbreak could provide a turning spot for this method of the rehabilitation area that has struggled to gain widespread acceptance.

\section{$\rightarrow$ What this article adds:}

There are clear limitations to implantation of telerehabilitation programs. Responding to limitations is an essential part of planning a sustainable telerehabilitation program. 
(12, 13), better access to therapists for counseling (14), providing facilities for physicians to conduct examinations $(11,13)$, reducing medical care costs $(12,13)$, reducing the cost of patients transfer to medical centers $(12,15)$, increasing patient satisfaction (11), increasing public health (16), and creating a managed care environment in hospitals and medical centers (13). In the corona outbreak, telerehabilitation has the potential to benefit both the patients and therapists by decreasing the inconvenience, respecting social distancing, eliminating distance barriers, and decreasing the cost of unnecessary patient transfers, and reducing travel time (17). Moreover, due to social distancing and dealing with a group of high-risk patients, implementation of telerehabilitation services is spiking in the world. On the other hand, for people not infected with the COVID-19 virus, especially those at higher risk of being affected (eg, older adults with pre-existing medical conditions, such as hypertension and diabetes mellitus), telerehabilitation can provide a convenient access to routine care without the risk of exposure in a congested hospital or medical practice waiting rooms.

\section{Previous Use of Telerehabilitation in Emergency Outbreaks}

Although telerehabilitation has been emerging for some time, and previously experts have warned that a new global pandemic is inevitable and could cycle over many months and might result in high absenteeism and quarantine (18), there is no documented paper for using telerehabilitation in an emergency outbreak. Some articles are found in scientific databases that address the role of telerehabilitation in neurologic and respiratory conditions. Telerehabilitation is primarily applied to physiotherapy (19), and neurorehabilitation for monitoring the rehabilitative progress of stroke patients (20). A study by Vasilopoulou et al shows home-based maintenance telerehabilitation reduces the risk for acute exacerbations of COPD, hospitalizations, and emergency department visits (21). In another article, Azhari and Parsa emphasized the importance of home-based rehabilitation in orthopedic surgery in COVID-19 Outbreak (22). It is believed that home rehabilitation may have similar effects on out-patient clinics and there is no difference between pediatric and adult patients (23).

\section{Telerehabilitation during COVID-19}

Three main groups benefit from telerehabilitation services in a coronavirus pandemic:

- The first groups are patients or even healthy people who have been quarantined at home to prevent infection. Under normal circumstances, these patients with stroke, musculoskeletal disorder, or cancer might be able to manage their neuromusculoskeletal conditions by leaving home and attending rehabilitation clinics. Nonetheless, people with older age, cardiovascular disease, diabetes, chronic respiratory disease, hypertension, and cancer are at a high risk during the COVID-19 pandemic (24).

Also, according to the recommendation of the World Confederation of Physiotherapy, all members should, as far as possible, postpone unnecessary treatment of their patients for safety reasons (25). Nevertheless, the confederation introduced telerehabilitation as an alternative service for 2 reasons: the decline in physiotherapists' incomes and the inconsistency of this recommendation with professional ethics; and patients' need for treatment (26). Telerehabilitation services that can be provided to these patients can be in the form of self-care recommendations, avoidance of inactivity, exercise therapy in the form of balance exercises concerning the individual's underlying diseases, range of motion exercises, strength exercises aimed at maintaining muscle mass, and breathing exercises to maintain the normal respiratory capacity of the person.

- The second group of people with COVID-19 are hospitalized. Patients admitted with COVID-19 commonly present with dyspnea, cough, hypoxia, fatigue, arthralgia, and myalgia, which may limit their overall functional ability and make the situation worse (27). Since the recommendations of the Center for Disease Control and Prevention are to reduce the physical exposure of therapists and patients as much as possible, telerehabilitation, especially breathing exercises that aimed at improving lung volume and capacity, training in proper breathing patterns, proper positioning, and cough hygiene can be helpful. Physiotherapist-patient consultations could be either synchronous or asynchronous.

Moreover, because some of the rehabilitation therapists working in the hospital are at high risk of developing the disease and are excluded from the service cycle of patients with COVID-19 according to the guidelines, they can reduce their workload through telerehabilitation.

- The third group are patients who have just been discharged from the hospital, but sometimes because of the limited capacity of hospitals, the entire rehabilitation program has not been fully implemented for them and the patient has not yet achieved independence before the illness. Delivering patient education, and providing a home exercise program (HEP) are an important part of the outpatient rehabilitation program (28). Telerehabilitation can help the patient by eliminating the physical distance and the need for the patient to return to medical centers while his immune system may be weak. Telerehabilitation can also be used to closely follow up patients (28).

\section{Obstacles, Barriers, and Recommendations}

Despite the surprising scenario, there are clear limitations to telerehabilitation programs. The needs assessment is a key part of planning a sustainable telerehabilitation program. This is vital for all careers and their needs must be broadly defined. An efficient telerehabilitation system can be developed if it is accepted by the consumers and used as a new and alternative method. We can categorize limitations to accessing the telerehabilitation in 4 factors:

Human Attitudes: Like all treatment options, emphasis will be given to the importanice of human factors in the planning, development, and implementing telerehabilitation systems and programs (29). Attitude plays an important role in our lives, thoughts, and individual and social behaviors. Our view on various issues determines our actions and reactions and justify our motivation or reluc- 
tance to show certain behaviors; and attitude toward telerehabilitation is not an exception. Patient motivation and acceptance is crucial for success. For example, one of the limitations is the separation of the physiotherapist-patient relationship. Many physical therapy, occupational therapy, audiology, optometry, and speech therapy assessments are dependent on expensive instruments. Also, in the treatment phase, the patients must perform the interventions correctly. Sometimes in cardiac and respiratory rehabilitation, vital sign monitoring are serious (30). Even though the development of telerehabilitation has led to the strengthening of the relationship between the therapist and the patient, in some cases due to the patients' disabilities, such as hearing or vision impairment or speech, communication is associated with problems that require knowledge-based companies to respond to the needs (31). Creating and developing software with the ability to communicate with the disabled and the elderly, by emphasizing the remaining visual, auditory, and tactile skills that are consistent with user abilities, can help solve this problem. Based on the results of the clinician's experience and global trends, it can be anticipated that in case of implementation of this technology in the field of rehabilitation, an overall positive trend in its acceptance and application by experts can be expected.

Technology: A remarkable increase in the number of patients treated by telerehabilitation is noticeable since 2002 to 2004 . After a subsequent decrease, the number of patients assisted by telerehabilitation increased starting from 2007, probably due to the support of new technologies and the overcoming of the initial skepticism to which every new technology is subjected (32). To better manage, analyze, and exchange information during the corona crisis and other expected crises, systems must reduce the burden of information management responsibility. They should facilitate the rapid recovery and retrieval of notes, categorization and rapid reporting of findings, and easy and timely access to new texts, databases, and knowledge. The growing efficiency of the internet, the vast global network, virtual reality, the construction of very small computers, and new content will advance the management of resources and scientific research on unforeseen events and the administration of affairs (33). The rapid growth of the internet use has made it useful to have access to hardware and computer software, and best of all, access to all places, due to user-compatible capabilities. Therapists and patients can now exchange audio, video images, texts, and required software for the internet. This progress can be further expanded.

Policy and Ethics: Confidentiality and privacy are an important part of the treatment process for patients who explain their most personal information to therapists (34). The basic principle of medical ethics, which should be considered in this method, is to take the patients' satisfaction in maintaining their information by the therapist. However, in circumstances in which cyberspace uses a database abroad, its possibility to hack its data any time may not provide a suitable place to observe these ethical principles. Morality is not limited to the therapist, but even the patients are responsible for considering the treatment advice specifically and avoiding disseminating information and treatments to others. Professional commitment and failure to introduce a therapist with fake titles is another dark spot that needs more attention from the judiciary. In order to access all of the patients' health information, such as blood tests, imaging, previous treatments, and patient history, the electronic health record available to all certified therapists must be uploaded to national websites.

Financial Issues and Standards: This treatment method requires its own time and skills, but currently these services are not mainly covered by insurance. Also, in case of error from the therapist, and the issues which are raised in the legal authorities, it is not clear how to deal with the case. According to the above-mentioned considerations, in the CTP book, some codes have been defined for this service, but the exact standard of how to execute these codes has not been written and implemented (35). Sometimes the weakness in the implementation of electronic health records and telerehabilitation is not due to technological problems but it is the consequence of wrong organizational culture and resistance of users.

\section{Conclusion}

Telerehabilitation has been steadily on the rise for years, but the corona virus brought it into a new light. During the COVID-19 pandemic, telerehabilitation offers rehabilitation practices, the ability to continue caring for patients, both those with and without COVID-19. However, this condition is not the last one. There are some limitations for implementation of telerehabilitation in all areas. Some aspects of rehabilitation, especially the assessment of the cardiopulmonary system, supervising the exercise and responses of patients in all conditions and application of modalities cannot be done remotely. Also, technology, rules, and therapists' training must be adapted to this approach. In general, 4 factors of the motivation of therapists, the existence of appropriate technical structure, ongoing investment, and technical support contribute to the success of remote rehabilitation. An important aspect of the future success of telerehabilitation involves proper training of people involved in these new forms of intervention. Educational systems should consider reeducation courses to suggest appropriate education to the therapist (36). On the other hand, insurance organizations should redesign their rules to support financial needs. They should pay attention to the fact that telerehabilitation is not a cost because it prevents and decreases severe diseases and surgeries (37). Telerehabilitation has the capacity to compensate heterogeneity in access to facilities and in establishing equity in health care. Finally, identifying its ethical challenges in various fields, including satisfaction, confidentiality, privacy, professional medical commitments, and anticipating the necessary arrangements in this area, is very important and requires special attention from the authorities (38). Although the use of rehabilitation is not yet widespread enough in the health system, it is an area where its expansion and adaptation are inevitable. Therefore, it is worthwhile for decision-makers in the field of health, treatment, and medical education to prepare 
themselves for rational encountering.

\section{Acknowledgment}

The authors wish to extend their gratitude to Shahid Beheshti University of Medical Sciences for financial support and sponsorship.

\section{Conflict of Interests}

The authors declare that they have no competing interests.

\section{References}

1. Khan S, Siddique R, Ali A, Xue M, Nabi G. Novel coronavirus, poor quarantine, and the risk of pandemic. J Hosp Infect. 2020 Feb 11.

2.Hassan SA, Sheikh FN, Jamal S, Ezeh JK, Akhtar A. Coronavirus (COVID-19): a review of clinical features, diagnosis, and treatment. Cureus. 2020 Mar;12(3).

3. Cucinotta D, Vanelli M. WHO declares COVID-19 a pandemic. Acta Biomed. 2020 Mar 19;91(1):157-60.

4. Sanche S, Lin YT, Xu C, Romero-Severson E, Hengartner NW, Ke $\mathrm{R}$. The novel coronavirus, 2019-nCoV, is highly contagious and more infectious than initially estimated. arXiv preprint arXiv. 2002.03268. 2020 Feb 9.

5. Gourinchas PO. Flattening the pandemic and recession curves. Mitigating the COVID Economic Crisis: Act Fast and Do Whatever. 2020;31:57-62.

6. Thomas P, Baldwin C, Bissett B, Boden I, Gosselink R, Granger CL, Hodgson C, Jones AY, Kho ME, Moses R, Ntoumenopoulos G. Physiotherapy management for COVID-19 in the acute hospital setting: clinical practice recommendations. J Physiother. 2020 Mar 30.

7. Ryu S. History of telemedicine: evolution, context, and transformation. Healthc Inform Res. 2010 Mar 1;16(1):65-6.

8. Ihrig C. Travel Cost Savings and Practicality for Low-Vision Telerehabilitation. Telemed J E Health. 2019 Jul 1;25(7):649-54.

9. Telemedicine-Opportunities and developments in member states [Internet] 2nd ed. Geneva, Switzerland: WHO press; 2010. Available from: https://www.who.int/goe/publications

10. Peretti A, Amenta F, Tayebati SK, Nittari G, Mahdi SS. Telerehabilitation: Review of the State-of-the-Art and Areas of Application. JMIR Rehabil Assist Technol. 2017;4(2):e7.

11. Bergmo TS. Economic evaluation in telemedicine-still room for improvement. J Telemed Telecare. 2010 Jul;16(5):229-31.

12. Nuara A, Fabbri-Destro M, Scalona E, Lenzi SE, Rizzolatti G, Avanzini P. Telerehabilitation in response to constrained physical distance: An opportunity to rethink neurorehabilitative routines. J Neurol. 2021 Jan 15:1-2.

13. Appleby E, Gill ST, Hayes LK, Walker TL, Walsh M, Kumar S. Effectiveness of telerehabilitation in the management of adults with stroke: a systematic review. PloS One. 2019 Nov 12;14(11):e0225150.

14. Dorstyn D, Mathias J, Denson L. Applications of telecounselling in spinal cord injury rehabilitation: a systematic review with effect sizes. Clin rehabil. 2013 Dec;27(12):1072-83.

15. Wade VA, Karnon J, Elshaug AG, Hiller JE. A systematic review of economic analyses of telehealth services using real time video communication. BMC Health Serv Res. 2010 Dec;10(1):1-3.

16. Marzano G, Lubkina V. Usability in social telerehabilitation systems for elderly users. Public health. 2017 Mar 1;144:1-3.

17. Movahedazarhouligh S, Vameghi R, Hatamizadeh N, Bakhshi E, Mousavi Khatat SM. The level of awareness of rehabilitation professionals employed in rehabilitation academic centers regarding tele-rehabilitation technology. Iran Rehabil J. 2015 Jun 10;13(2):5761.

18. Li J, Seale H, Ray P, Rawlinson W, Lewis L, MacIntyre CR. Issues regarding the implementation of eHealth: Preparing for future influenza pandemics. Interact J Med Res. 2012;1(2):e20.

19. Mani S, Sharma S, Omar B, Paungmali A, Joseph L. Validity and reliability of Internet-based physiotherapy assessment for musculoskeletal disorders: a systematic review. J Telemed Telecare. 2016 Mar 31.
20. Jagos H, David V, Haller M, Kotzian S, Hofmann M, Schlossarek S, Eichholzer K, Winkler M, Frohner M, Reichel M, Mayr W, Rafolt D. A framework for (tele-) monitoring of the rehabilitation progress in stroke patients: eHealth 2015 special issue. Appl Clin Inform. 2015;6(4):757-68.

21. Vasilopoulou M, Papaioannou AI, Kaltsakas G, Louvaris Z, Chynkiamis N, Spetsioti S, Kortianou E, Genimata SA, Palamidas A, Kostikas K, Koulouris NG. Home-based maintenance telerehabilitation reduces the risk for acute exacerbations of COPD, hospitalisations and emergency department visits. Eur Respir J. 2017 May 1;49(5).

22. Azhari A, Parsa A. Covid-19 Outbreak Highlights: Importance of Home-Based Rehabilitation in Orthopedic Surgery. Arch Bone Jt Surg. 2020 Apr 1;8(Covid-19 Special Issue):317-8.

23. Mehta SP, Roy JS. Systematic review of home physiotherapy after hip fracture surgery. J Rehabil Med. 2011;43(6):477-80.

24. $\mathrm{Wu} \mathrm{Z,} \mathrm{McGoogan} \mathrm{JM.} \mathrm{Characteristics} \mathrm{of} \mathrm{and} \mathrm{important} \mathrm{lessons}$ from the coronavirus disease 2019 (COVID-19) outbreak in China: summary of a report of 72314 cases from the Chinese Center for Disease Control and Prevention. JAMA. 2020 Apr 7;323(13):123942.

25. World Confederation for Physical Therapy. Information and resources about COVID-19. https://www.wcpt.org/news/NovelCoronavirus-2019-nCoV. 2020. Accessed April 23rd2020.

26. World Confederation for Physical Therapy. Report sets out future of digital physical therapy practice. https://www.wcpt.org/news/report-sets-out-future-of-digitalphysical-therapy-practice. 2020. Accessed April 23rd2020.

27. Paolino G, Canti V, Santo Raffaele Mercuri PR, Candiani M, Pasi F. Diffuse cutaneous manifestation in a new mother with COVID-19 (SARS-Cov-2). Int J Dermatol. 2020 May 2.

28. Arzani P, Khalkhali Zavieh M, Khademi-Kalantari K, Akbarzadeh Baghban A. Pulmonary rehabilitation and exercise therapy in a patient with COVID-19: A Case report. Med J Islam Repub Iran. 2020;34(1):731-734.

29. Tsai JM, Cheng MJ, Tsai HH, Hung SW, Chen YL. Acceptance and resistance of telehealth: The perspective of dual-factor concepts in technology adoption. Int J Inf Manage. 2019 Dec 1;49:34-44.

30. Filos D, Triantafyllidis A, Chouvvarda I, Buys R, Cornelissen VA, Budts W, Walsh D, Woods C, Moran K, Maglaveras N. PATHway: Decision Support in Exercise Programmes for Cardiac Rehabilitation. Stud Health Technol Inform. 2016 Jun 16 (pp. 4045).

31. Mahmoudi E, Meade MA. Disparities in access to health care among adults with physical disabilities: analysis of a representative national sample for a ten-year period. Disabil Health J. 2015 Apr $1 ; 8(2): 182-90$.

32. Peretti A, Amenta F, Tayebati SK, Nittari G, Mahdi SS. Telerehabilitation: review of the state-of-the-art and areas of application. JMIR Rehabil Assist Technol. 2017;4(2):e7.

33. Van Dyk L. A review of tielehealth service implementation frameworks. Int J Environ Res Public Health. 2014 Feb;11(2):127998.

34. Richmond T, Peterson C, Cason J, Billings M, Terrell EA, Lee AC, Towey M, Parmanto B, Saptono A, Cohn ER, Brennan D. American Telemedicine Association's principles for delivering telerehabilitation services. Int J Telerehabil. 2017;9(2):63.

35. Bali S. Barriers to Development of Telemedicine in Developing Countries. InTelehealth. 2018 Diec 5. IntechOpen. Accessed June $23,2020$.

36. Rhone K, Lindgren J, Mack LJ, Nelson ME, Spencer L, Anderson SM. Avera eCARE: Medical Student Education in Telemedicine. Telehealth Med Today. 2020 Apr 30.

37. Lykke F, Holzworth M, Rosager M, Turisco F. Telemedicine: An essential technology for reformed health care. Falls Church, Virginia: CSC Insights: Computer Sciences Corporation. 2013.

38. Langarizadeh M, Moghbeli F, Aliabadi A. Application of ethics for providing telemedicine services and information technology. Med Arch. 2017 Oct;71(5):351. 